\title{
Foot-floor contact pattern in children and adults with Dravet Syndrome
}

\section{Authors:}

Lore Wyers ${ }^{1}$, MSc, Research Group MOVANT, Department of Rehabilitation Sciences and Physiotherapy (REVAKI), University of Antwerp, Wilrijk, Belgium; Multidisciplinary Motor Centre Antwerp, University of Antwerp, Belgium

Roberto Di Marco ${ }^{1}, \mathrm{PhD}$, Department of Neuroscience, Section of Rehabilitation, Laboratory of Clinical Analysis and Biomechanics of Movement and Posture NEUROMOVE-Rehab, University of Padova, Padova, Italy

Stefano Zambelli, BSc, Department of Information Engineering, University of Padova, Padova, Italy; Department of Neuroscience, Section of Rehabilitation, Laboratory of Clinical Analysis and Biomechanics of Movement and Posture NEUROMOVE-Rehab, University of Padova, Padova, Italy

Stefano Masiero, MD, Department of Neuroscience, Section of Rehabilitation, Laboratory of Clinical Analysis and Biomechanics of Movement and Posture NEUROMOVE-Rehab, University of Padova, Padova, Italy; PNC, Padova Neuroscience Center, Padova, Italy

Ann Hallemans*, PhD, Research Group MOVANT, Department of Rehabilitation Sciences and Physiotherapy (REVAKI), University of Antwerp, Wilrijk, Belgium; Multidisciplinary Motor Centre Antwerp, University of Antwerp, Belgium

Patricia Van de Walle, PT, PhD, Research Group MOVANT, Department of Rehabilitation Sciences and Physiotherapy (REVAKI), University of Antwerp, Wilrijk, Belgium; Multidisciplinary Motor Centre Antwerp, University of Antwerp, Belgium

Kaat Desloovere, PhD, Department of Rehabilitation Sciences, KU Leuven, Leuven, Belgium; Clinical Motion Analysis Laboratory, University Hospital Leuven, Pellenberg, Belgium

Alessandra Del Felice, MD, PhD, Department of Neuroscience, Section of Rehabilitation, Laboratory of Clinical Analysis and Biomechanics of Movement and Posture NEUROMOVE-Rehab, University of Padova, Padova, Italy; PNC, Padova Neuroscience Center, Padova, Italy

${ }^{1}$ Lore Wyers and Roberto Di Marco were equally responsible for the work described in this paper.

* Corresponding author at: Movant, Universiteitsplein 1, Campus Drie Eiken, D.S.022, 2610, Wilrijk, Belgium. E-mail address: ann.hallemans@uantwerpen.be (A. Hallemans).

\section{Abstract}

Background: Dravet Syndrome (DS) is a developmental and epileptic encephalopathy characterized by severe drug-resistant seizures and associated with cognitive and motor impairments. Walking problems are frequently observed. As the foot plays a key role during walking, compromised foot function can be a feature of deviant gait. 
Aim: To investigate foot function in DS by characterizing foot-floor contact patterns using pedobarography.

Methods: A total of 31 children and adults were included in the DS group (aged 5.2-32.8 years, 17 female, 174 steps) and 30 in the control group (aged 6.0-32.9, 16 female, 180 steps). The foot-floor contact pattern was evaluated based on progression, length and smoothness (spectral arc length) of the center of pressure (CoP). Linear mixed models were used to identify differences between non-heel strikes and heel strikes and between the DS and control group.

Results: Fifteen participants with DS showed inconsistency in the type of foot-floor contact (heel strikes and non-heel strikes). Heel strikes of participants with DS had significantly reduced time of CoP under the hindfoot and increased time under the midfoot region compared to the control group. Significant time and age effects were detected.

Conclusions and Implications: Deviant foot-floor contact patterns were observed in DS. Possible gait immaturity and instability as well as implications for interventions are discussed.

\section{Keywords}

Severe Myoclonic Epilepsy of Infancy (SMEI), gait, pedobarography, biomechanics, center of pressure, heel strike

\section{Highlights}

- Center of pressure trajectories were used to assess foot function in Dravet Syndrome

- Half of the patients did not consistently perform heel strikes

- Non-heel striking affects length, progression and smoothness of CoP trajectories

- Inconsistent heel strikers show quick transition of $\mathrm{CoP}$ from hind- to midfoot

\section{Introduction}

Dravet Syndrome (DS) is a developmental and epileptic encephalopathy with an estimated prevalence between $1 / 15000$ and $1 / 40000$. In at least $80 \%$ of cases, it is caused by mutations in the gene encoding the sodium channel type I alpha subunit, SCNIA [1,2]. The syndrome is characterized by drug resistant infantile onset seizures accompanied by cognitive, behavioral and motor impairments $[3,4]$. Children with DS show a delay in motor development before the age of two, often with a delayed achievement of independent walking [5-7]. Diverse gait alterations have been described in DS, with about half of the investigated cases presenting a gait pattern which resembles crouch gait $[6,8,9]$. Although gait problems are a major concern for children and adults with DS and their caregivers [10,11], scarce literature has addressed this topic and especially quantitative assessments of gait deviations are lacking [8].

The foot plays a key role during walking as it supports the weight of the body on the ground. The dynamically functioning foot is required to be sufficiently flexible to provide stability on any surface 
and at the same time to be a rigid lever able to transmit propulsive forces for an efficient walking pattern [12]. Detailed evaluation of foot function is possible using three dimensional motion analysis with multisegment foot models [13-15]. However, this procedure requires high levels of participant collaboration, which makes it a less feasible option in DS due to cognitive and behavioral problems. Less complex approaches such as pedobarography may provide meaningful insight into foot function. In pedobarography, pressure platforms are used to analyze the pressure distribution under the foot. Quantitative measures enable the objective detection of disturbed foot-floor contact patterns [16].

A relevant plantar pressure measure is the center of pressure $(\mathrm{CoP})$ and its trajectory on the plantar surface, also referred to as 'gait line'. The CoP is defined as the centroid of all external forces acting on the plantar surface of the foot and is often used as an indirect measure of neuromuscular control $[17,18]$. A mature foot-floor contact pattern is characterized by a heel strike at initial contact followed by a fluent movement of the CoP from the medial aspect of the heel over the lateral side of the foot, ending with a quick medial shift on the forefoot. Impaired motor control may affect the ability to consistently perform heel strikes. Deviations in CoP trajectories may reflect compromised foot function and pathological gait [18]. This study aims to investigate foot function in children and adults with DS by characterizing footfloor contact patterns using pedobarography. We hypothesize that differences in progression, length and smoothness of the CoP trajectory can be detected between patients with DS who consistently perform heel strikes, those who are not able to consistently perform heel strikes and able-bodied controls.

\section{Methods}

\subsection{Setting}

Data collection was performed at the Laboratory of Clinical Analysis and Biomechanics of Movement and Posture, University Hospital of Padua, Italy. Patients with DS were recruited at Neurological Institute Carlo Besta, Milan, Italy, University Hospital of Padua, Italy and Verona University Hospital, Verona, Italy. Measurements took place between May 2015 and October 2019. The study was approved by the Ethical Committee of the Padua University Hospital (protocol number 4276/AO/17).

\subsection{Inclusion}

All participants with a minimum age of five years and a genetically confirmed diagnosis of DS were eligible. Exclusion criteria were the inability to walk without assistance, or the occurrence of a convulsive seizure within 24 hours prior to the examination. Age-matched able-bodied volunteers were enrolled as control group. Exclusion criteria for the control group were a history of neurological or orthopedic disorders. All participants and their legal guardians provided written informed consent.

\subsection{Data collection}

Height, weight and foot length (from the most proximal apex of calcaneus to the most distal apex of the toes) were measured and inspection of the foot posture in stance was performed. Pedobarographic data 
were collected using the midgait method [19] on a walkway with an embedded plantar pressure platform $\left(100 \mathrm{~Hz}, 4\right.$ sensors $/ \mathrm{cm}^{2}$, from 10 to $12720 \mathrm{kPa}, 47.5 \times 32.0 \mathrm{~cm}^{2}$, max force: $193 \mathrm{kN}$, emed-q®, Novel $\mathrm{GmbH}$, Munich, Germany). Participants walked barefoot at self-selected walking velocity and were instructed not to look down or target the pressure mat. As long as participant cooperation was ensured, trials were repeated until at least six successful steps per side were collected. In case participants were less cooperative, only the side(s) with a minimum of three successful steps were included. Due to behavioral issues, all 'clean' steps on the platform were collected, even steps that were less representative for the participant's usual gait pattern. Afterwards, steps considered most representative were identified in a standardized manner. Hereto, custom made MATLAB ${ }^{\circledR}$ scripts (R2018a, The Mathworks Inc, Natick, MA, US) were used to calculate correlations between all pressure distribution images within each participant (appendix A). For each side, the three footprints with the highest correlation among each other were selected for further analysis.

\subsection{Processing}

The longitudinal axis of the foot was determined as the bisect between the tangents for the medial and lateral sides of the maximum pressure picture. Three regions were identified based on two lines perpendicular to the longitudinal axis as defined in the platform's standard software: one at $27 \%$ of the heel-toe distance to separate hindfoot and midfoot, and one at 55\% to separate midfoot and forefoot (figure 1c). The toes were included in the forefoot region (Multimask v23 Novel GmbH, Munich, Germany). Manual quality control assured correct masking and confirmed that all included steps were full footprints. The CoP was determined as the weighted centroid of the total number of active sensors for each data sample. Based on the region where the first CoP point was situated, steps were identified as heel strikes (hindfoot) or non-heel strikes (mid- or forefoot region). A low pass fourth order Butterworth filter at $20 \mathrm{~Hz}$ was applied to the CoP trajectory over time, using a reflection technique to avoid edge effects at the beginning and end of the signal [20]. After filtering, six parameters were calculated as presented in table 1 . Filtering and parameter calculations were implemented in MATLAB®.

The CoP progression (CoPP) was calculated for the three foot regions as the time during which CoP was situated under that region relative to total contact time (TCT). Length ratio was defined as the CoP trajectory path length normalized by clinically measured foot length. Mature foot-floor contact patterns were expected to have a length ratio of approximately one, with larger values indicating less efficient gait due to additional or compensatory movements. Smoothness of the CoP trajectory was assessed by calculating the spectral arc length (SPARC), the arc length of the Fourier magnitude spectrum within an adaptive frequency range. This smoothness index proved to be sensitive, robust to measurement noise and independent of temporal movement scaling [21]. SPARC was calculated for the medio-lateral (ML SPARC) and antero-posterior (A-P SPARC) components of the CoP displacement vector, using code 
provided by Balasubramanian et al. (2015) [21]. SPARC is a negative number with larger absolute values indicating more interruptions and thus less smooth $\mathrm{CoP}$ trajectories.

\subsection{Statistical analysis}

Descriptive statistics were computed for demographics and step characteristics. The demographic data were compared between groups using Mann-Whitney U tests, after Shapiro-Wilk tests did not confirm a normal distribution.

Non-heel strikes were only observed in the DS group and resulted in v-shaped CoP trajectories (figure 1a). Because of this different shape, it would not have been appropriate to compare them with heel strikes from the control group. We therefore used a two-stage approach. In the first stage, the effect of the type of initial contact on CoP parameters was analyzed within the DS group and non-heel strikes were omitted in stage two. In the second stage, distinction was made between participants with DS that always performed heel strikes and those who inconsistently switched between non-heel strikes and heel strikes. Comparison of CoP parameters was then performed between the two DS subgroups ('DS_consistent' and 'DS_inconsistent') and the control group.

Linear mixed models were fitted to assess the effect of the type of initial contact (non-heel strike or heel strike) and group (DS_consistent, DS_inconsistent or control) respectively, on the main outcome parameters. Age, TCT and side (left, right) were added as fixed effects. To account for nonindependence of observations, subject and side, nested within subject, were entered as random effects. Significance of the fixed effects was tested using a likelihood ratio test. We first fitted linear mixed models testing the effect of type of initial contact on each of the six outcome measures within the DS group. Subsequently, we fitted linear mixed models to detect differences between the DS subgroups and control group for all outcome parameters. If the existence of a significant difference was revealed, post hoc pairwise comparisons (Bonferroni) using estimated marginal means, were performed to detect differences between groups. All statistical analyses were performed in R (v 4.0.0, R Foundation, Vienna, Austria, packages 'Ime4' and 'emmeans') [22]. Details on the linear mixed models analysis can be found in appendix B.

The nominal significance level was set at $P<.05$, but may not be appropriate as a cut-off for the linear mixed models, because multiple hypotheses were tested. Since there were moderate correlations between the six outcomes, a Bonferroni correction would be too conservative. A Benjamini-Hochberg correction, controlling for a false discovery rate of 0.05 , was applied to interpret the results of the linear mixed models [23]. 


\section{Results}

\subsection{Demographics and step characteristics}

In total, 31 children and adults with DS (174 steps) and 30 age-matched typically developing volunteers (180 steps) with an age range from 5.2 to 32.9 years were included in the study (table 2). Out of 46 eligible candidates with DS, 15 patients needed to hold hands for guidance or support, could not repeat trials until a sufficient number of steps were collected or did not walk in a straight line in a representative manner and were thus excluded. Among the 31 participants with DS, 17 had planovalgus feet (pronation) and four hindfoot varus (supination), of whom 12 wore insoles with arch and hindfoot support or orthopedic shoes (table 2).

Out of a total of 174 steps in the DS group, 139 (80\%) were heel strikes and 34 (20\%) were non-heel strikes (eight with first $\mathrm{CoP}$ on the midfoot region and 27 on the forefoot region). The non-heel strikes were observed in 16 participants with DS of which one always performed forefoot strikes while the others varied with heel strikes. These 16 participants formed the DS_inconsistent subgroup with a median age of 11.1 years (range 5.2 - 24.6 years). The 15 participants with DS who always performed heel strikes, formed the DS_consistent subgroup with a median age of 15.1 years (range $6.9-32.8$ years). The DS_inconsistent subgroup was significantly younger $(P=.03)$ with lower height $(P=.03)$ than the DS_consistent subgroup.

\subsection{Stage 1: Comparison of non-heel strikes with heel strikes within participants with DS}

Linear mixed models compared heel strikes with non-heel strikes within the DS group, accounting for age and total contact time (TCT). The likelihood ratio test $\left(\chi^{2}\right)$ revealed a significant difference between the two types of initial contact for all parameters except CoPP midfoot and M-L SPARC. When controlling for age and TCT, the CoPP hindfoot was significantly shorter $(P<.001)$, while CoPP forefoot was longer $(P<.001)$ in case of a non-heel strike, with increased Length Ratio $(P<.001)$ and decreased smoothness on A-P SPARC ( $P<.001$; figure 2). Detailed statistical output is reported in appendix B.

One outlier with a length ratio of 2.50 was observed among the non-heel strikes in the DS group. This outlier did not alter the conclusions on the effect of type of initial contact.

\subsection{Stage 2: Comparison of heel strikes between DS subgroups and typically developing volunteers}

When comparing heel strikes between the DS subgroups and control group, accounting for TCT and age, the likelihood ratio test $\left(\chi^{2}\right)$ detected a significant difference for CoPP hindfoot $(P=.002)$ and CoPP midfoot $(P<.001)$. Post hoc pairwise comparison revealed decreased CoPP hindfoot $(P=.003)$ and increased CoPP midfoot $(P<.001)$ in the DS_inconsistent subgroup compared controls. Other parameters did not differ significantly (figure 2). Detailed statistical output is reported in appendix B. 


\subsection{Relation with TCT and age}

In addition to group effects, the linear mixed models investigated the effects of TCT and age on the different CoP trajectory parameters. In stage 1 , TCT had a significant effect on all CoP trajectory parameters, while age did not show any significant effect. With increasing TCT, an increase of CoPP hindfoot $(P=.03)$, CoPP midfoot $(P=.002)$ and Length Ratio $(P=.03)$ was observed and a decrease of CoPP forefoot $(P<.001)$, M-L SPARC $(P<.001)$ and A-P SPARC $(P<.001)$. In stage 2 , significant effects of age and TCT were observed. With increasing TCT, CoPP midfoot slightly increased and CoPP forefoot, M-L SPARC and A-P SPARC decreased (all $P<.001$ ). Small but significant age effects revealed decreased CoPP hindfoot $(P=.04)$, increased CoPP forefoot $(P=.01)$ and reduced M-L SPARC $(P<.001)$ with increasing age. Details on linear mixed models and the resulting regression equations can be found in appendix B.

\section{Discussion}

This study aimed to investigate foot function by characterizing the foot-floor contact pattern in a group of people with DS compared to a group of typically developing children and able-bodied adults. Six parameters were selected to evaluate the progression, length and smoothness of the CoP trajectories on the plantar surface of the foot. About half of the participants with DS did not consistently initiate their step with a heel strike. The relative duration of CoP progression in non-heel strikes was larger on the forefoot and lower on the hindfoot, as was expected since most of the non-heel strikes were forefoot strikes. The typically v-shaped CoP trajectory of these non-heel strikes was reflected in longer path lengths and decreased antero-posterior smoothness. The DS_inconsistent subgroup also showed deviant CoP trajectories when only heel strikes were compared with DS_consistent and control group. More specifically, the CoP progression significantly differed, revealing a quick transition of the CoP from hind- to midfoot region in DS_inconsistent. Contrary to our hypothesis, DS participants did not perform heel strikes with different path lengths or smoothness compared to the control group. These results showed that the foot-floor contact pattern was significantly different in patients who were unable to consistently perform heel strikes. Patients with impaired foot function can be distinguished based on foot strike pattern and deviant CoP trajectories.

In previous research on typically developing children, mid- and forefoot strikes have been frequently observed early after the onset of independent walking. The foot strike patterns normalized to heel strikes during the first year of walking experience and the gait line appeared smoother with increasing age (Bertsch, Unger, Winkelmann, \& Rosenbaum, 2004; Gallahue \& Ozmun, 1998; Hallemans, De Clercq, Dongen, \& Aerts, 2006). In DS, not only a later onset of independent walking but also a persisting delay in overall motor development is observed [5], which could contribute to immature foot strike patterns. These observations might indicate immaturity of gait in children and adults with DS. This hypothesis is 
supported by the findings of significant age effects on CoP trajectory parameters and younger age in the DS_inconsistent group.

Even when participants with DS did perform heel strikes, it is likely that increased time during which CoP was situated on the midfoot (increased CoPP midfoot) bears the functional significance of a quest for more stability or may reflect altered muscle function. Stability issues during gait are in line with previous research suggesting that atypical crouch gait and increased step width were compensation strategies to increase stability by lowering the center of mass and widening the base of support [9]. Impaired motor control or muscle strength may cause the incapacity to slow down the forward motion of the tibia [27] which would result in a quick transition of the CoP from hind- to midfoot. This may be reflected in kinematic deviations previously described in this population, specifically early and increased ankle dorsiflexion [9] and might contribute to crouch gait [6,9,27]. Pathophysiologic findings in DS suggest that motor control is often disturbed owing to central nervous disorders [28,29] and possibly peripheral neuropathy [7]. Clear muscle weakness is not reported, but studies evaluating strength are lacking [6]. Future studies should investigate the link between CoP progression, clinical findings and the gait pattern.

Besides stability, strength or control issues, also malalignment of the foot may affect the excursion of the CoP [17]. Deviating static foot postures were observed in $65 \%$ of the participants with DS, mostly pronated feet (planovalgus). Foot morphology could directly affect the CoP trajectory and may also be linked to abnormal foot strike patterns, as $77 \%$ of the participants with pronated feet also performed non-heel strikes. Further investigation of the dynamic behavior of these deviations during gait and the influence of supportive footwear is needed to clarify how foot morphology may contribute to gait deviations in children and adolescents with DS.

For in depth investigation of the link between CoP trajectories and the gait pattern, additional video or three dimensional gait analysis (3DGA) is required. This combination proved to be more accurate to identify regions of the foot [30] and to detect the absence of a heel strike [31]. Furthermore, 3DGA provides essential information on gait patterns and should ideally be performed to assess gait in patients with DS. However, even better participant collaboration is needed to collect reliable 3DGA data and it requires advanced motion capture infrastructure [32]. Hence, when 3DGA proves too demanding in participants with severe cognitive and behavioral impairments, pedobarography combined with video analysis may offer a feasible alternative. The $\mathrm{CoP}$ trajectory may then provide insight into foot function and mechanical control to enable integrated interpretation of observed gait deviations [18].

The results imply that people with DS may benefit from interventions that improve foot-floor contact patterns and provide stability in order to walk with a more efficient pattern. Physiotherapy with an emphasis on practicing correct foot strike patterns and improving plantar flexor muscle function could 
be indicated. Shoes could provide stability around ankle and foot, especially in combination with orthopedic insoles in case of foot deformities.

Cognitive and behavioral impairments in people with DS were a limiting factor that could impact the generalizability of the results. Out of 46 eligible candidates, 15 were excluded because the minimum of three representative steps for one side was not reached owing to behavioral or cognitive limitations. It remains unclear whether these participants' foot-floor contact patterns were similar to the included participants with DS or if their exclusion resulted in an underrepresentation of severely affected patients in this study.

\subsection{Conclusion}

Half of the participants with DS showed inconsistency in the type of foot-floor contact (heel strikes and non-heel strikes). Even when participants in this subgroup performed heel strikes, their CoP progression pattern differed from typically developing controls, more specifically by a reduced duration of CoP under the heel and a prolonged duration under the midfoot.

\section{Acknowledgements and funding}

The authors thank the participants and their families for their cooperation and dr. Erik Fransen for his statistical expertise. This study was supported by Dravet Italia ONLUS (Associazione Italiana Sindrome di Dravet Onlus), Gruppo Famiglie Dravet, and YouReCa KU Leuven. The authors declare that they have no conflict of interest.

\section{References}

[1] L. Claes, J. Del-Favero, B. Ceulemans, L. Lagae, C. Van Broeckhoven, P. De Jonghe, De novo mutations in the sodium-channel gene SCN1A cause severe myoclonic epilepsy of infancy, Am. J. Hum. Genet. (2001). doi: $10.1086 / 320609$.

[2] A. Brunklaus, R. Ellis, E. Reavey, G.H. Forbes, S.M. Zuberi, Prognostic, clinical and demographic features in SCN1A mutation-positive Dravet syndrome, Brain. 135 (2012) 2329-2336. doi:10.1093/brain/aws151.

[3] C. Dravet, The core Dravet syndrome phenotype, Epilepsia. 52 (2011) 3-9. doi:10.1111/j.1528-1167.2011.02994.x.

[4] I.E. Scheffer, S.F. Berkovic, G. Capovilla, M.B. Connolly, J.A. French, L. Guilhoto, E. Hirsch, S. Jain, G.W. Mathern, S.L. Moshé, D.R. Nordli, E. Perucca, T. Tomson, ILAE classification of the epilepsies: Position paper of the ILAE Commission for Classification and Terminology, Epilepsia. 58 (2017) 512-521. doi:10.1111/epi.13709.

[5] K. Verheyen, E. Verbecque, B. Ceulemans, A.-S. Schoonjans, P. Van De Walle, A. Hallemans, Motor development in children with Dravet syndrome, Dev. Med. Child Neurol. 61 (2019) 950-956. doi:10.1111/dmcn.14147.

[6] J.M. Rodda, I.E. Scheffer, J.M. McMahon, S.F. Berkovic, K. Graham, Progressive gait deterioration in adolescents with Dravet syndrome, Arch. Neurol. 69 (2012) 873-878. doi:10.1001/archneurol.2011.3275.

[7] C. Gitiaux, N. Chemaly, S. Quijano-Roy, C. Barnerias, I. Desguerre, M. Hully, C. Chiron, O. Dulac, R. Nabbout, Motor neuropathy contributes to crouching in patients with Dravet syndrome, Neurology. 87 (2016) $277-281$. doi:10.1212/WNL.0000000000002859.

[8] L. Wyers, P. Van de Walle, A. Hoornweg, I.T. Bobescu, K. Verheyen, B. Ceulemans, A.-S. Schoonjans, K. Desloovere, A. Hallemans, J.M. Rodda, I. Tepes Bobescu, Gait deviations in patients with dravet syndrome: A systematic review, Eur. J. Paediatr. Neurol. 23 (2019) 357-367. doi:10.1016/j.ejpn.2019.03.003.

[9] R. Di Marco, A. Hallemans, G. Bellon, F. Ragona, E. Piazza, T. Granata, B. Ceulemans, A.-S. Schoonjans, P. Van de 
Walle, F. Darra, B. Dalla Bernardina, M. Vecchi, Z. Sawacha, B. Scarpa, S. Masiero, M.G. Benedetti, A. Del Felice, Gait abnormalities in people with Dravet syndrome: A cross-sectional multi-center study, Eur. J. Paediatr. Neurol. 23 (2019) 808-818. doi:10.1016/j.ejpn.2019.09.010.

[10] P. Camfield, C. Camfield, K. Nolan, Helping Families Cope with the Severe Stress of Dravet Syndrome, Can. J. Neurol. Sci. 43 (2016) S9-S12. doi:10.1017/cjn.2016.248.

[11] N. Villas, M.A. Meskis, S. Goodliffe, Dravet syndrome: Characteristics, comorbidities, and caregiver concerns, Epilepsy Behav. 74 (2017) 81-86. doi:10.1016/j.yebeh.2017.06.031.

[12] J.S. Bevans, Biomechanics: a review of foot function in gait, Foot. 2 (1992) 79-82. doi:10.1016/0958-2592(92)90022H.

[13] K. Deschamps, F. Staes, P. Roosen, F. Nobels, K. Desloovere, H. Bruyninckx, G.A. Matricali, Body of evidence supporting the clinical use of 3D multisegment foot models: A systematic review, Gait Posture. 33 (2011) 338-349. doi:10.1016/j.gaitpost.2010.12.018.

[14] A. Leardini, P. Caravaggi, T. Theologis, J. Stebbins, Multi-segment foot models and their use in clinical populations, Gait Posture. 69 (2019) 50-59. doi:10.1016/j.gaitpost.2019.01.022.

[15] R. Di Marco, S. Rossi, V. Racic, P. Cappa, C. Mazzà, Concurrent repeatability and reproducibility analyses of four marker placement protocols for the foot-ankle complex, J. Biomech. 49 (2016) 3168-3176. doi:10.1016/j.jbiomech.2016.07.041.

[16] K. Deschamps, P. Roosen, F. Nobels, P.-A. Deleu, I. Birch, K. Desloovere, H. Bruyninckx, G.A. Matricali, F. Staes, Review of clinical approaches and diagnostic quantities used in pedobarograpic measurements, J Sport. Med Phys Fit. 55 (2015) 191-204.

[17] E.A. Fuller, Center of pressure and its theoretical relationship to foot pathology., J. Am. Podiatr. Med. Assoc. 89 (1999) 278-291. doi:10.7547/87507315-89-6-278.

[18] E.G. Jameson, J.R. Davids, J.P. Anderson, R.B. Davis, D.W. Blackhurst, L.M. Christopher, Dynamic pedobarography for children: Use of the center of pressure progression, J. Pediatr. Orthop. 28 (2008) 254-258. doi:10.1097/BPO.0b013e318164ee6e.

[19] T.G. McPoil, M.W. Cornwall, L. Dupuis, M. Cornwell, Variability of plantar pressure data: A comparison of the twostep and midgait methods, J. Am. Podiatr. Med. Assoc. 89 (1999) 495-501. doi:10.7547/87507315-89-10-495.

[20] A. De Cock, J. Vanrenterghem, T. Willems, E. Witvrouw, D. De Clercq, The trajectory of the centre of pressure during barefoot running as a potential measure for foot function, Gait Posture. 27 (2008) 669-675. doi:10.1016/j.gaitpost.2007.08.013.

[21] S. Balasubramanian, A. Melendez-Calderon, A. Roby-Brami, E. Burdet, On the analysis of movement smoothness, J. Neuroeng. Rehabil. 12 (2015) 1-11. doi:10.1186/s12984-015-0090-9.

[22] D. Bates, M. Mächler, B.M. Bolker, S.C. Walker, Fitting linear mixed-effects models using lme4, J. Stat. Softw. 67 (2015). doi:10.18637/jss.v067.i01.

[23] Y. Benjamini, Y. Hochberg, Controlling the False Discovery Rate : A Practical and Powerful Approach to Multiple Testing, 57 (1995) 289-300. https://www.jstor.org/stable/2346101.

[24] A. Hallemans, D. De Clercq, S. Van Dongen, P. Aerts, Changes in foot-function parameters during the first 5 months after the onset of independent walking: A longitudinal follow-up study, Gait Posture. 23 (2006) 142-148. doi:10.1016/j.gaitpost.2005.01.003.

[25] C. Bertsch, H. Unger, W. Winkelmann, D. Rosenbaum, Evaluation of early walking patterns from plantar pressure distribution measurements. First year results of 42 children, Gait Posture. 19 (2004) 235-242. doi:10.1016/S09666362(03)00064-X.

[26] D.L. Gallahue, J.C. Ozmun, Fundamental Movement Abilities, in: Underst. Mot. Dev. Infants, Child. Adolesc. Adults, 4th ed., McGraw-Hill, Boston, Massachusetts, 1998: p. 541.

[27] J.R. Gage, Gait Analysis; an essential tool in the treatment of Cerebral Palsy, Clin. Orthop. Relat. Res. 288 (1993) 126-134.

[28] W.A. Catterall, Dravet syndrome: a sodium channel interneuronopathy, Curr. Opin. Physiol. 2 (2018) 42-50. doi:10.1016/j.cophys.2017.12.007.

[29] F. Darra, D. Battaglia, C. Dravet, M. Patrini, F. Offredi, D. Chieffo, E. Piazza, E. Fontana, G.G. Olivieri, I. Turrini, B. Dalla Bernardina, T. Granata, F. Ragona, Dravet syndrome: Early electroclinical findings and long-term outcome in adolescents and adults, Epilepsia. 60 (2019) S49-S58. doi:10.1111/epi.16297. 
[30] C. Giacomozzi, J.A. Stebbins, Anatomical masking of pressure footprints based on the Oxford Foot Model: validation and clinical relevance, Gait Posture. 53 (2017) 131-138. doi:10.1016/j.gaitpost.2016.12.022.

[31] A.J. Mudge, M. Sangeux, E.A. Wojciechowski, M.G. Louey, M.J. McKay, J.N. Baldwin, L.N. Dwan, M.W. Axt, J. Burns, Can pedobarography predict the occurrence of heel rocker in children with lower limb spasticity?, Clin. Biomech. 71 (2019) 208-213. doi:10.1016/j.clinbiomech.2019.10.022.

[32] A. Hallemans, P. Van de Walle, L. Wyers, K. Verheyen, A.-S. Schoonjans, K. Desloovere, B. Ceulemans, Clinical usefulness and challenges of instrumented motion analysis in patients with intellectual disabilities, Gait Posture. 71 (2019) 105-115. doi:10.1016/j.gaitpost.2019.04.016. 


\section{Tables and figures}

\begin{tabular}{|l|l|l|}
\hline CoP trajectory & Parameter & Definition \\
\hline Progression & CoPP hindfoot & CoP progression on hindfoot $(\%$ of total contact time) \\
CoPP midfoot & $\begin{array}{l}\text { CoP progression on midfoot (\% of total contact time) } \\
\text { CoP progression on forefoot }(\% \text { of total contact time) }\end{array}$ \\
\hline Length & Length Ratio & CoP trajectory path length $(\mathrm{cm}) /$ foot length $(\mathrm{cm})$ \\
\hline Smoothness & M-L SPARC & Medio - lateral spectral arc length \\
& A-P SPARC & Antero - posterior spectral arc length \\
\hline
\end{tabular}

Table 1: Definition of the main outcome variables. CoPP,center of pressure progression; CoP, center of pressure; SPARC, Spectral arc length; M-L, medio-lateral; A-P, antero-posterior.

\begin{tabular}{|c|c|c|c|c|}
\hline \multirow[b]{2}{*}{ Demographics } & \multicolumn{3}{|c|}{ DS } & \multirow{2}{*}{$\begin{array}{l}\text { Control } \\
(\mathbf{n}=\mathbf{3 0})\end{array}$} \\
\hline & $\begin{array}{c}\text { Total } \\
(\mathbf{n}=\mathbf{3 1})\end{array}$ & $\begin{array}{c}\text { DS_consistent } \\
\text { subgroup } \\
(\mathbf{n}=15)\end{array}$ & $\begin{array}{c}\text { DS_inconsistent } \\
\text { subgroup } \\
(n=16)\end{array}$ & \\
\hline Age (year) & $13.0(5.2 ; 32.8)$ & $15.1(6.9 ; 32.8)^{*}$ & $11.1(5.2 ; 24.6)^{*}$ & $13.5(6.0 ; 32.9)$ \\
\hline Gender (male/female) & $14 / 17$ & $7 / 8$ & $7 / 9$ & $14 / 16$ \\
\hline Height (cm) & $147.0(106.0 ; 194.5)$ & $155.5(119.5 ; 194.5)^{*}$ & $139.8(106.0 ; 164.0)^{*}$ & $160.5(117.0 ; 180.5)$ \\
\hline Weight (kg) & $42.0(18.5 ; 83.5)$ & $51.0(20.0 ; 83.5)$ & $35.0(18.5 ; 69.5)$ & $48.5(19.5 ; 81.5)$ \\
\hline \multicolumn{5}{|l|}{ Foot morphology (n): } \\
\hline Pronation (planovalgus) & 17 & 6 & 11 & \\
\hline Supination (varus) & 4 & 3 & 1 & \\
\hline Neutral & 10 & 6 & 4 & \\
\hline Insoles or orthopedic shoes (n) & 12 & 5 & 7 & \\
\hline
\end{tabular}

Table 2: Demographics per group and subgroup. Median (min; max); * significant difference between DS subgroups. DS, Dravet Syndrome; n, number of participants. 


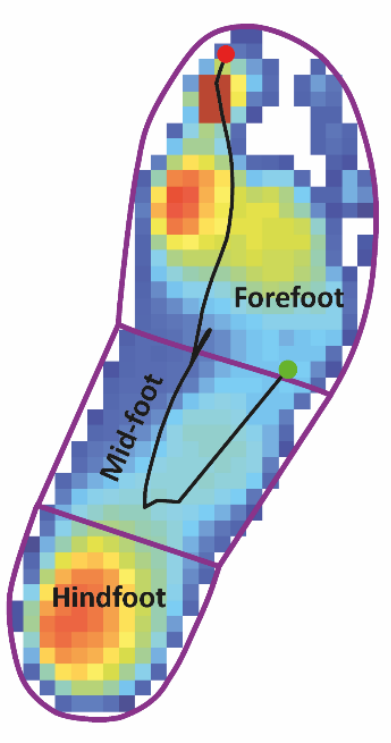

(a)

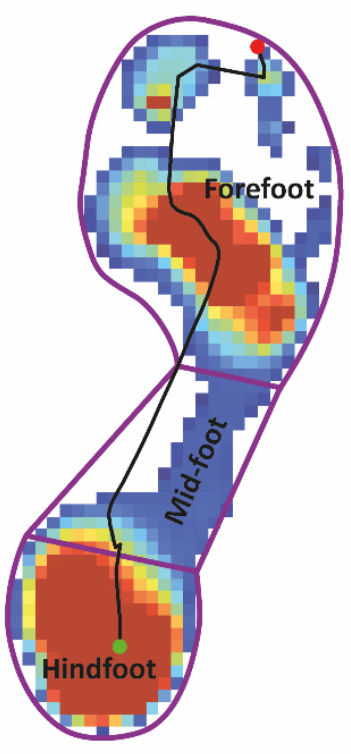

(b)

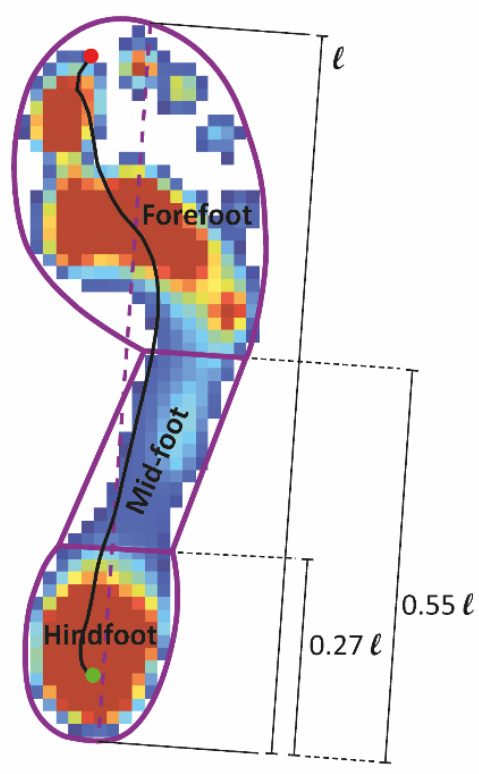

(c)

Figure 1: Example images of center of pressure trajectories on maximal pressure images of a) non-heel strike by a participant with DS, b) heel strike by a participant with DS and c) heel strike by a typically developing volunteer.

Purple lines indicates the applied masking to identify the hind-, mid- and forefoot regions, with those being separated by two lines perpendicular to the longitudinal axis of the footprint (purple dashed line in (c): one at $27 \%$ of the heel-toe distance $(\boldsymbol{\ell})$ to separate hindfoot and midfoot; and one at $55 \%$ to separate midfoot and forefoot. 

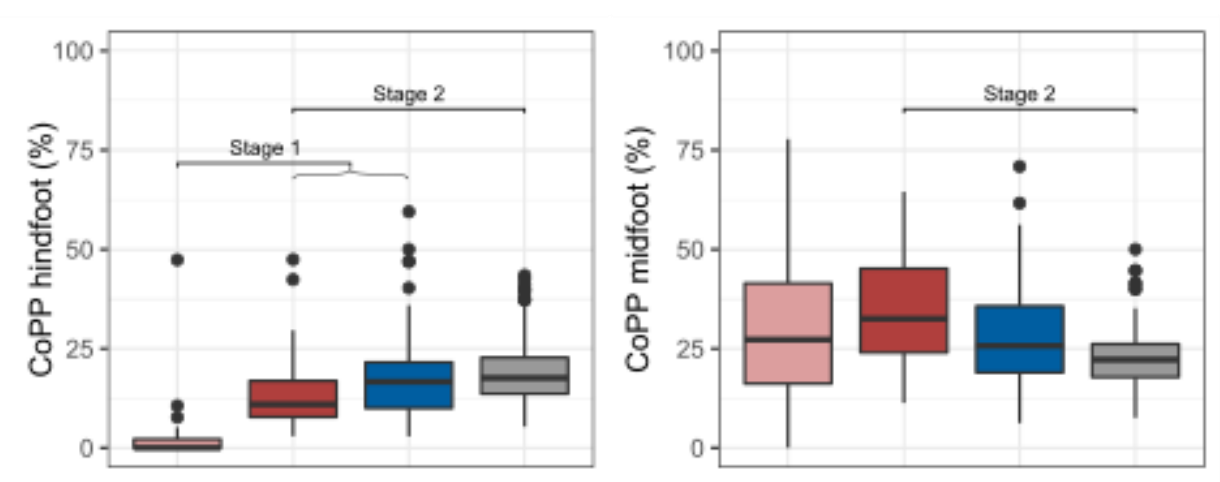

DS_inconsistent, non-heel strikes

DS_inconsistent, heel strikes

DS_consistent

Control
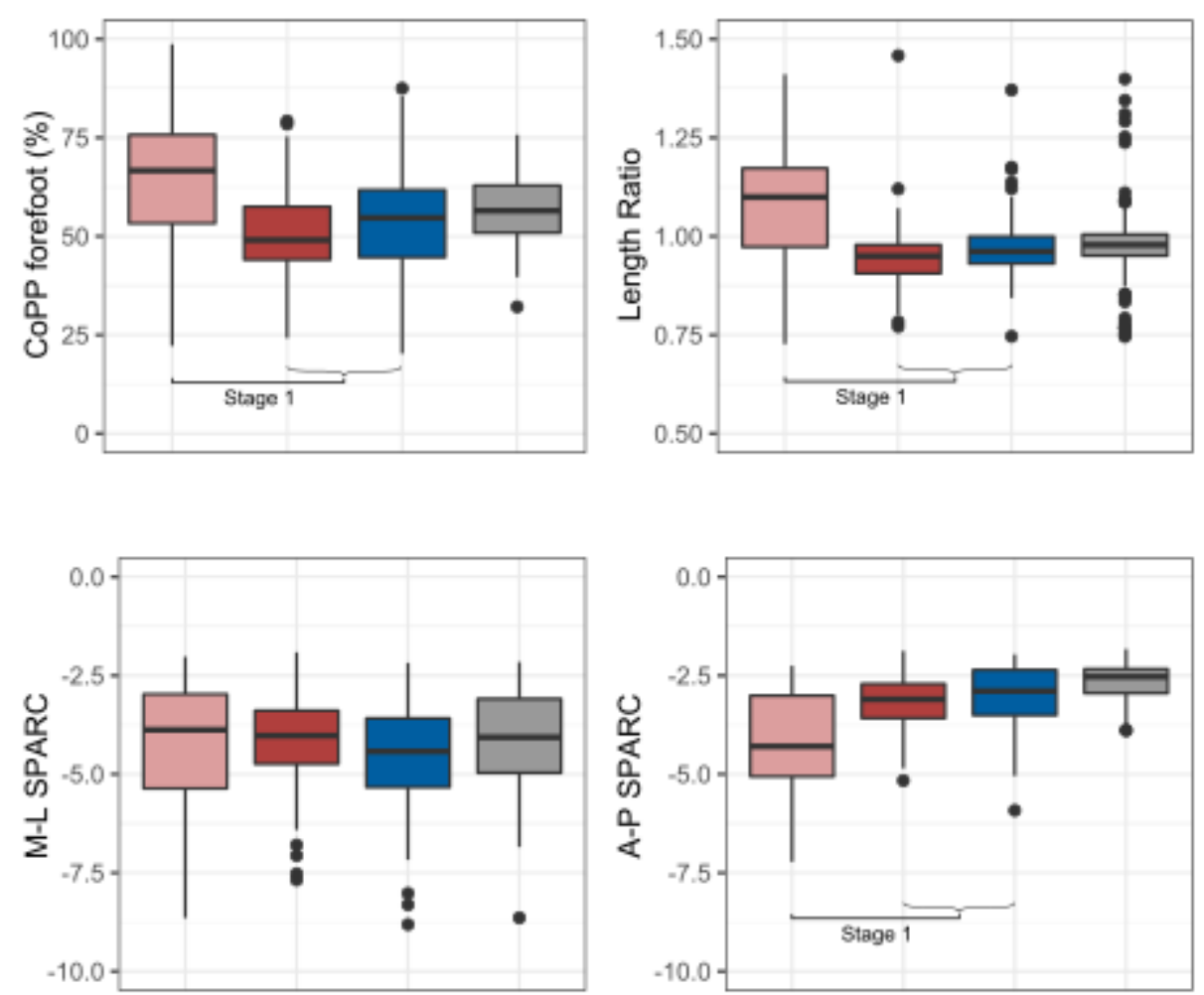

Statistical analysis using LMM Stage 1: $\square$ vs $\square+\square$ Stage 2: $\square$ vs $\square$ vs $\square$

Figure 2: CoP trajectory parameters per (sub)group with subdivision of heel strikes and non-heel strikes. Significant differences where detected using linear mixed models (LMM) controlling for age and total contact time in two stages: between non-heel strikes and heel strikes within DS (Stage 1) and between heel strikes of DS_inconsistent, DS_consistent and control group (Stage 2). DS, Dravet Syndrome; CoPP, center of pressure progression; SPARC, Spectral arc length; M-L, medio-lateral; A-P, antero-posterior. 


\section{APPENDIX A: Correlation matrix calculation}

As long as participants cooperation was ensured, trials were repeated until at least six successful steps per side were collected. Due to behavioral difficulties, all 'clean' steps on the platform were collected, even steps that were less representative for the participants usual gait pattern. Less representative altered walking patterns such as playful 'funny' walking, pauses on the force plate or direction changes, would be reflected in the $2 D$ pressure image of the footprint. Steps that were considered as the most representative were identified in a standardized manner. Hereto, custom made MATLAB® scripts (R2018a, The Mathworks Inc, Natick, MA, US) were used to calculate correlations between all pressure distribution images within each participant. For each side, the three footprints with the highest correlation among each other were selected for further analysis.

Each footprint was originally exported in ASCII-files, containing values measured by each sensor identified via row and columns coordinates describing the whole device. The area containing the footprint (region of interest, ROI) was then extracted without ignoring null areas possibly associated with cavus feet. ROI boundaries were identified as the first and last non-null rows and columns of the device-matrix.

Different footprints could have been differently oriented with respect to the device reference frame due to: 1) Walking direction not perfectly longitudinal to the device; 2) Altered walking pattern. Different walking direction would only affect the footprint orientation, whereas an altered walking pattern may additionally affect the pressure values. Extracted ROIs were therefore reoriented by first fitting an ellipse containing the whole ROI, and then using the angle between the main axis of inertia of this ellipse and the horizontal axis to rotate the footprint.

The reoriented images, i.e. the pressure value matrices, are then pairwise compared via the CORR2 Matlab function. This function computes the Pearson's correlation coefficient $(r)$ between two matrices $\mathbf{A}$ and $\mathbf{B}$ of the same dimensions (in the case of images $\mathbf{A}$ and $\mathbf{B}$ are two matrices of pixel intensity). This 2D- $r$ is calculated similarly to the $1 \mathrm{D}$ case, and specifically:

$$
r=\frac{\sum_{i} \sum_{j}\left(a_{i j}-\overline{\bar{a}}\right)\left(b_{i j}-\overline{\bar{b}}\right)}{\sqrt{\left[\sum_{i} \sum_{j}\left(a_{i j}-\overline{\bar{a}}\right)^{2}\right]\left[\sum_{i} \sum_{j}\left(b_{i j}-\overline{\bar{b}}\right)^{2}\right]}}
$$

Where:

- $a_{i j}$ is the element of the matrix $\mathbf{A}$ at row $i$ and column $j$;

- $b_{i j}$ is the element of the matrix B at row $i$ and column $j$;

- $\quad \overline{\bar{a}}$ and $\overline{\bar{b}}$ are the grand average (i.e., over rows and columns) of the element values of the matrices $\mathbf{A}$ and $\mathbf{B}$, respectively. 
The function CORR2 calculates the difference between each $i j$-th value in A and B and the grand average of those whole matrices, with respect to the difference of each individual matrix.

As a result of the comparison of $n$ images, a symmetric square $n$-order correlation matrix is obtained as output of the algorithm (Figure A1), with each $x y$-th element being the Pearson's coefficient $r$ calculated comparing the $x$ and $y$ images

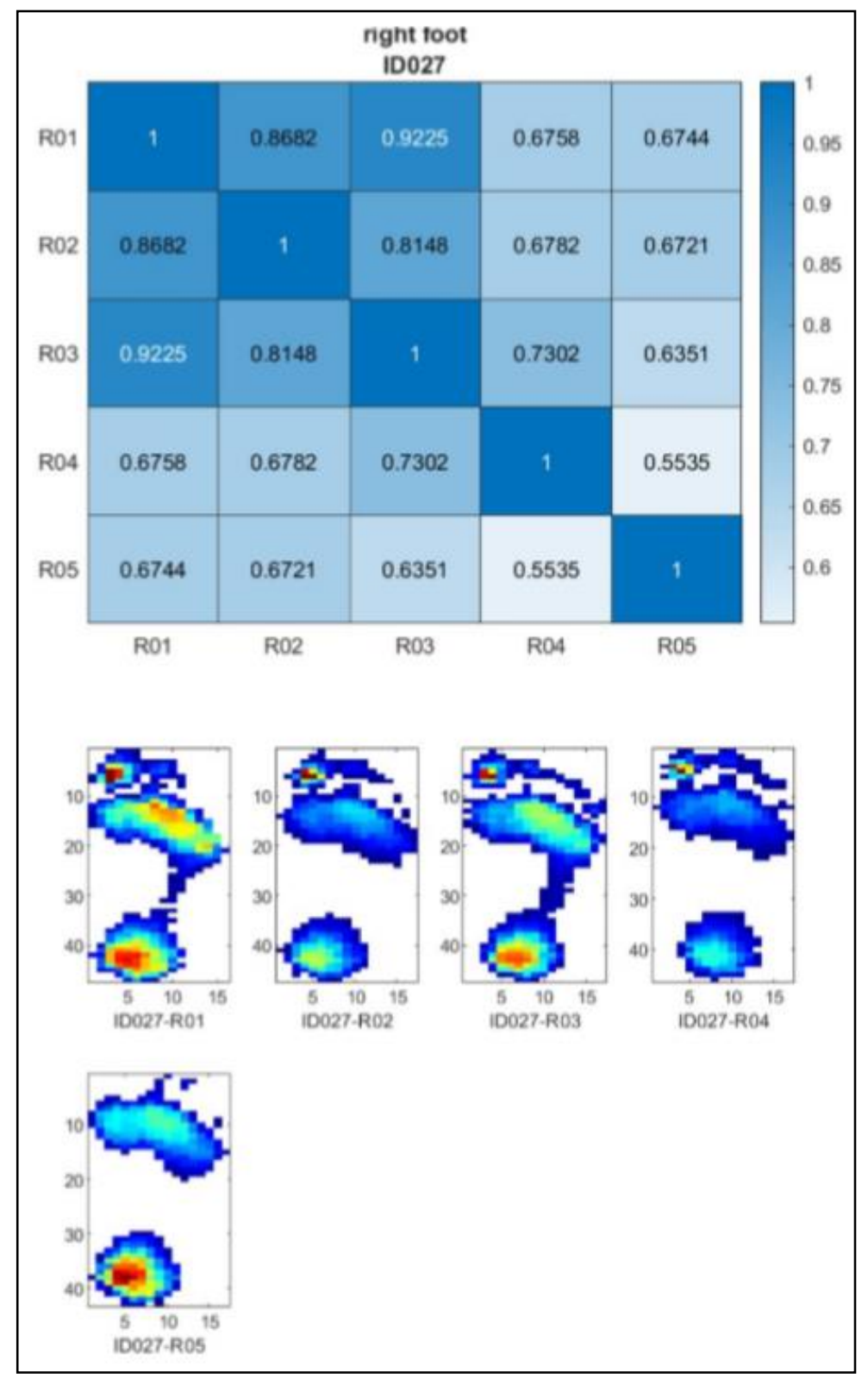

Figure A1 - An example of correlation matrix (above) obtained with the algorithm used to select the footprints (below). The main diagonal displays ones as the algorithm compares the i-th image with the image itself. 


\section{APPENDIX B: Linear mixed models analysis}

The difference in outcome between i) heel strikes and non-heel strikes and ii) Dravet Syndrome (DS) and control group, accounting for age and total contact time (TCT), was modeled using linear mixed models (LMM). LMM are a type of regression models that support the analysis of non-independent data. Where traditional regression and ANOVA techniques assume independent observations, LMM can account for the relatedness between observations by including random effect terms. These terms describe the dependence structure of the observations and the standard errors of the parameter estimates are adjusted accordingly.

In this particular case, multiple measurements were included per individual participant. Measurements within one participant were not independent. In addition, participants were repeatedly tested for the left side and the right side. Observations within one side were not independent either. This latter dependence was nested within the within-participant dependence.

Hence, LMM contain two types of independent variables. The variables for which the effect is to be tested (usually the research question of the experiment) are referred to as "fixed effects". In the current study they included group, age and TCT. The variables describing the dependence between observations are referred to as "random effects". In this study they included individual and side.

Two linear mixed models were fitted: the first one (Stage 1) within DS, with the fixed effect for group describing heel strikes versus non-heel strikes, and the second one (Stage 2) within heel strikes, with the fixed effect for group describing DS versus control. In addition to the fixed effect for group, we added the covariates age, total contact time (TCT) and side as fixed effects. Since TCT is directly related to walking velocity, this factor was added to account for velocity-dependent differences. Random effects for subject and side, nested within subject, were entered to account for non-independence of observations within individuals and sides. Significance of the fixed effects was tested using a likelihood ratio test.

For $i=1 \ldots n$, with $n$ the number of subjects and $j=1 \ldots m$, with $m$ the number of trials, the outcome parameter $Y_{i j}$ can be estimated using the following formula:

$$
Y_{i j}=\left(\beta_{0}+b_{i}\right)+\beta_{1} * \operatorname{group}_{i}+\beta_{2} * \operatorname{age}_{i}+\beta_{3} * T C T_{i j}+\beta_{4} * \operatorname{side}_{i j}+\varepsilon_{i j}
$$

with $\beta_{0}$ the population intercept, $b_{i}$ the individuals random deviation from the intercept, $\beta_{1}$ the fixed effect of group (with $\beta_{1 \_\mathrm{c}}$ the coefficient for the DS_consistent subgroup and $\beta_{1_{\_} \mathrm{i}}$ for the DS_inconsistent subgroup), $\beta_{2}$ the fixed effect of age, $\beta_{3}$ the fixed effect of total contact time, $\beta_{4}$ the fixed effect of side and $\varepsilon_{i j}$ the residuals. In stage 1, group was defined as heel strike (0) or non-heel strike (1). In stage 2, group was defined as control (0) or Dravet Syndrome (1). The estimated coefficients with their standard 
error (SE) and the $P$-value of the likelihood ratio test $\left(\chi^{2}\right)$ can be found in table B1 and LMM predictions are plotted for TCT in figures B1 and B2.

For two parameters (CoPP hindfoot and CoPP midfoot), a significant fixed effect for the variable 'group' existed. Post-hoc pairwise comparison with Bonferroni correction was performed calculating estimated marginal means, in order to assess the significance of the differences between pairs of subgroups. Results of the post hoc tests are reported in table B2.

\begin{tabular}{|c|c|c|c|c|c|c|c|c|c|c|c|}
\hline & \multicolumn{5}{|c|}{$\begin{array}{c}\text { Stage } 1 \\
\text { Non-heel strikes versus heel strikes (DS only) } \\
\end{array}$} & \multicolumn{6}{|c|}{$\begin{array}{c}\text { Stage } 2 \\
\text { DS subgroups versus control (heel strikes only) }\end{array}$} \\
\hline & $\beta_{0}$ & $\beta_{1}$ & $\beta_{2}$ & $\boldsymbol{\beta}_{3}$ & $\beta_{4}$ & $\beta_{0}$ & $\beta_{1 \_c}$ & $\beta_{1 \_\mathrm{i}}$ & $\boldsymbol{\beta}_{2}$ & $\beta_{3}$ & $\beta_{4}$ \\
\hline & \multicolumn{11}{|c|}{$Y_{i j}=$ CoPP hindfoot } \\
\hline Estimate & 0.091 & -0.115 & -0.002 & $1.37 \mathrm{E}-04$ & -0.003 & 0.181 & -0.019 & -0.075 & -0.002 & $8,24 \mathrm{E}-05$ & $-0,001$ \\
\hline SE & 0.049 & 0.020 & 0.002 & $6.27 \mathrm{E}-05$ & 0.013 & 0.038 & 0.020 & 0.021 & 0.001 & $5,82 \mathrm{E}-05$ & 0,009 \\
\hline \multirow[t]{2}{*}{$P$-value } & & $<.001 *$ & .22 & $.03 *$ & .83 & & \multicolumn{2}{|c|}{$.002 *$} & $.04^{*}$ & .17 & .92 \\
\hline & \multicolumn{11}{|c|}{$Y_{i j}=$ CoPP midfoot } \\
\hline Estimate & 0.155 & 0.006 & -0.002 & $2.50 \mathrm{E}-04$ & 0.008 & 0.106 & 0.027 & 0.100 & -0.001 & $2,27 \mathrm{E}-04$ & $-0,005$ \\
\hline SE & 0.070 & 0.027 & 0.003 & $8.21 \mathrm{E}-05$ & 0.019 & 0.043 & 0.023 & 0.024 & 0.001 & $6,65 \mathrm{E}-05$ & 0,011 \\
\hline \multirow[t]{2}{*}{$P$-value } & & .81 & .37 & $<.001 *$ & .67 & & \multicolumn{2}{|c|}{$<.001^{*}$} & .30 & $<.001 *$ & .63 \\
\hline & \multicolumn{11}{|c|}{$Y_{i j}=$ CoPP forefoot } \\
\hline Estimate & 0.754 & 0.089 & 0.003 & $-3.91 \mathrm{E}-04$ & -0.001 & 0.697 & -0.015 & -0.035 & 0.003 & $-3,04 \mathrm{E}-04$ & 0,011 \\
\hline SE & 0.065 & 0.026 & 0.002 & 7.95E-05 & 0.020 & 0.045 & 0.025 & 0.026 & 0.001 & $6,60 \mathrm{E}-05$ & 0,011 \\
\hline \multirow[t]{2}{*}{$P$-value } & & $<.001 *$ & .13 & $<.001 *$ & .98 & & \multicolumn{2}{|c|}{.38} & $.01 *$ & $<.001^{*}$ & .32 \\
\hline & \multicolumn{11}{|c|}{$Y_{i j}=$ Length Ratio } \\
\hline Estimate & 0.869 & 0.189 & -0.004 & $2.05 \mathrm{E}-04$ & 0.024 & 0.966 & -0.010 & -0.038 & -0.002 & $7,08 \mathrm{E}-05$ & 0,007 \\
\hline SE & 0.074 & 0.030 & 0.002 & $9.50 \mathrm{E}-05$ & 0.021 & 0.037 & 0.025 & 0.026 & 0.001 & $4,82 \mathrm{E}-05$ & 0,008 \\
\hline \multirow[t]{2}{*}{$P$-value } & & $<.001 *$ & .11 & $.03 *$ & .24 & & \multicolumn{2}{|c|}{.32} & .13 & .14 & .39 \\
\hline & \multicolumn{11}{|c|}{$Y_{i j}=$ M-L SPARC } \\
\hline Estimate & -1.331 & -0.500 & -0.038 & $-3.38 \mathrm{E}-03$ & 0.036 & $-1,668$ & 0.030 & -0.008 & -0.047 & -0.003 & 0,070 \\
\hline SE & 0.651 & 0.273 & 0.021 & 8.19E-04 & 0.218 & 0.491 & 0.252 & 0.268 & 0.014 & 0.001 & 0,132 \\
\hline \multirow[t]{2}{*}{$P$-value } & & .08 & .07 & $<.001 *$ & .86 & & \multicolumn{2}{|c|}{.98} & $<.001 *$ & $<.001 *$ & .60 \\
\hline & \multicolumn{11}{|c|}{$Y_{i j}=$ A-P SPARC } \\
\hline Estimate & -1.189 & -1.336 & 0.009 & $-2.84 \mathrm{E}-03$ & -0.027 & $-1,067$ & -0.064 & -0.295 & 0.003 & -0.003 & 0,078 \\
\hline SE & 0.416 & 0.160 & 0.016 & 4.94E-04 & 0.124 & 0.242 & 0.148 & 0.158 & 0.008 & $3,46 \mathrm{E}-04$ & 0,056 \\
\hline$P$-value & & $<.001 *$ & .56 & $<.001 *$ & .82 & & .1 & & .72 & $<.001^{*}$ & 0.16 \\
\hline
\end{tabular}

Table B1: Results of the linear mixed models and likelihood ratio test per parameter: * significant after Benjamini-Hochberg correction with false discovery rate 0.05; DE, Dravet Syndrome; SE, Standard Error; CoPP, center of pressure progression; SPARC, Spectral arc length; M-L, medio-lateral; A-P, anterio-posterior. 


\begin{tabular}{|l|ccc|}
\hline & $\begin{array}{c}\text { Control } \\
\text { versus } \\
\text { DS_consistent }\end{array}$ & $\begin{array}{c}\text { Control } \\
\text { versus } \\
\text { DS_inconsistent }\end{array}$ & $\begin{array}{c}\text { DS_consistent } \\
\text { versus } \\
\text { DS_inconsistent }\end{array}$ \\
\hline & \multicolumn{3}{|c|}{$\boldsymbol{Y}_{\boldsymbol{i} j}=$ CoPP hindfoot } \\
\hline Estimate & 0.019 & 0.075 & 0.056 \\
SE & 0.020 & 0.021 & 0.024 \\
$P$-value & 1.00 & $.003^{*}$ & .08 \\
\hline & & $\boldsymbol{Y}_{i j}=$ CoPP midfoot \\
\hline Estimate & -0.027 & -0.100 & -0.074 \\
SE & 0.023 & 0.025 & 0.028 \\
$P$-value & .77 & $<.001^{*}$ & .03 \\
\hline
\end{tabular}

Table B2: Results of the post hoc estimated marginal means analysis: pairwise differences of the variable 'group'. *significant at $\alpha=.05$ with Bonferroni correction; DS, Dravet syndrome subgroup of patients that always performed heel strikes (consistent) or patients that switched between heel strikes and non-heel strikes (inconsistent); SE, Standard Error; CoPP, center of pressure progression.
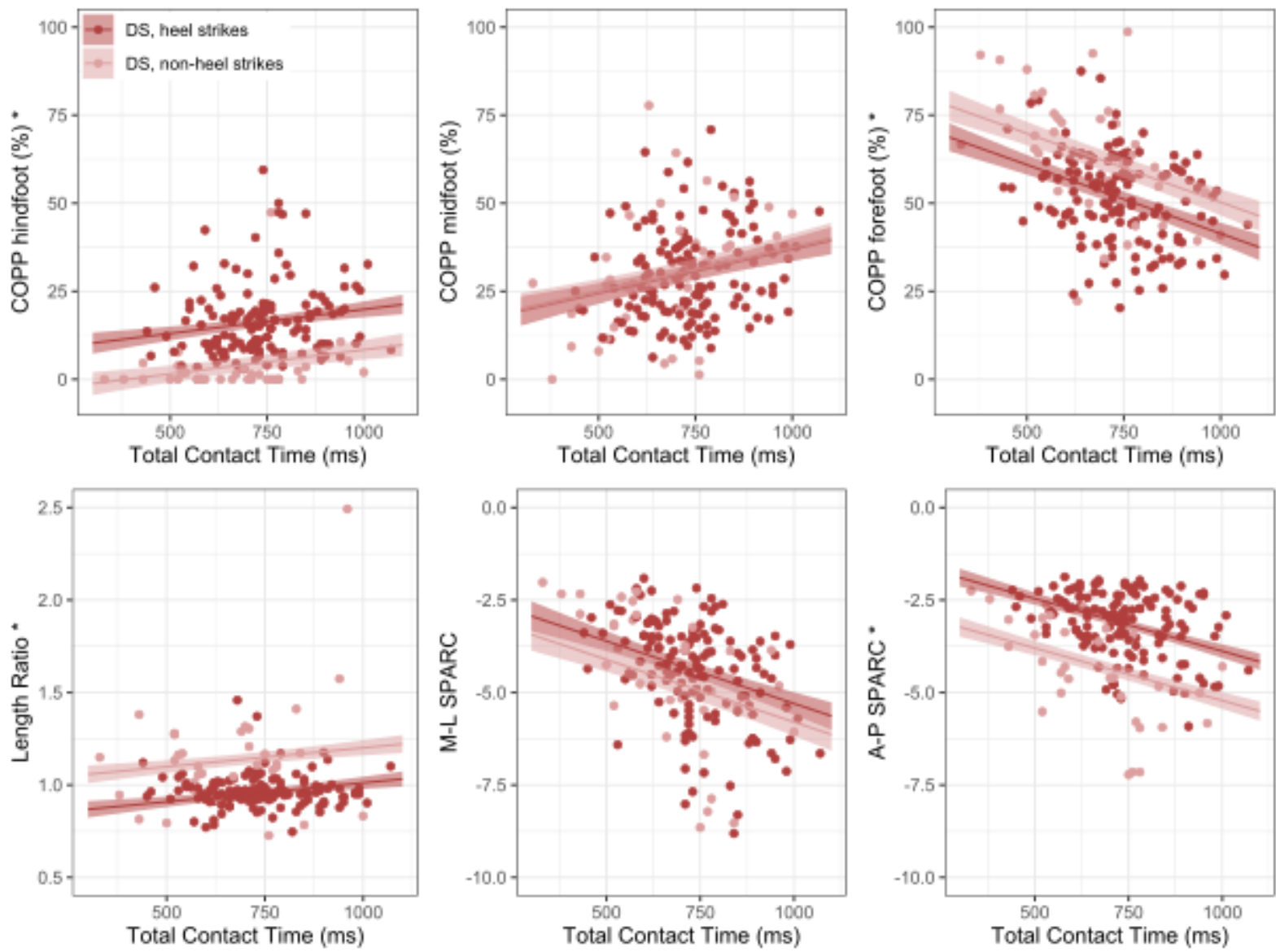

Figure B1: Linear mixed model predictions for total contact time within the Dravet Syndrome (DS) group. * Parameter with significant difference between heel strikes (dark red) and non-heel strikes (light red); CoPP, center of pressure progression; SPARC, Spectral arc length; M-L, medio-lateral; A-P, antero-posterior. 

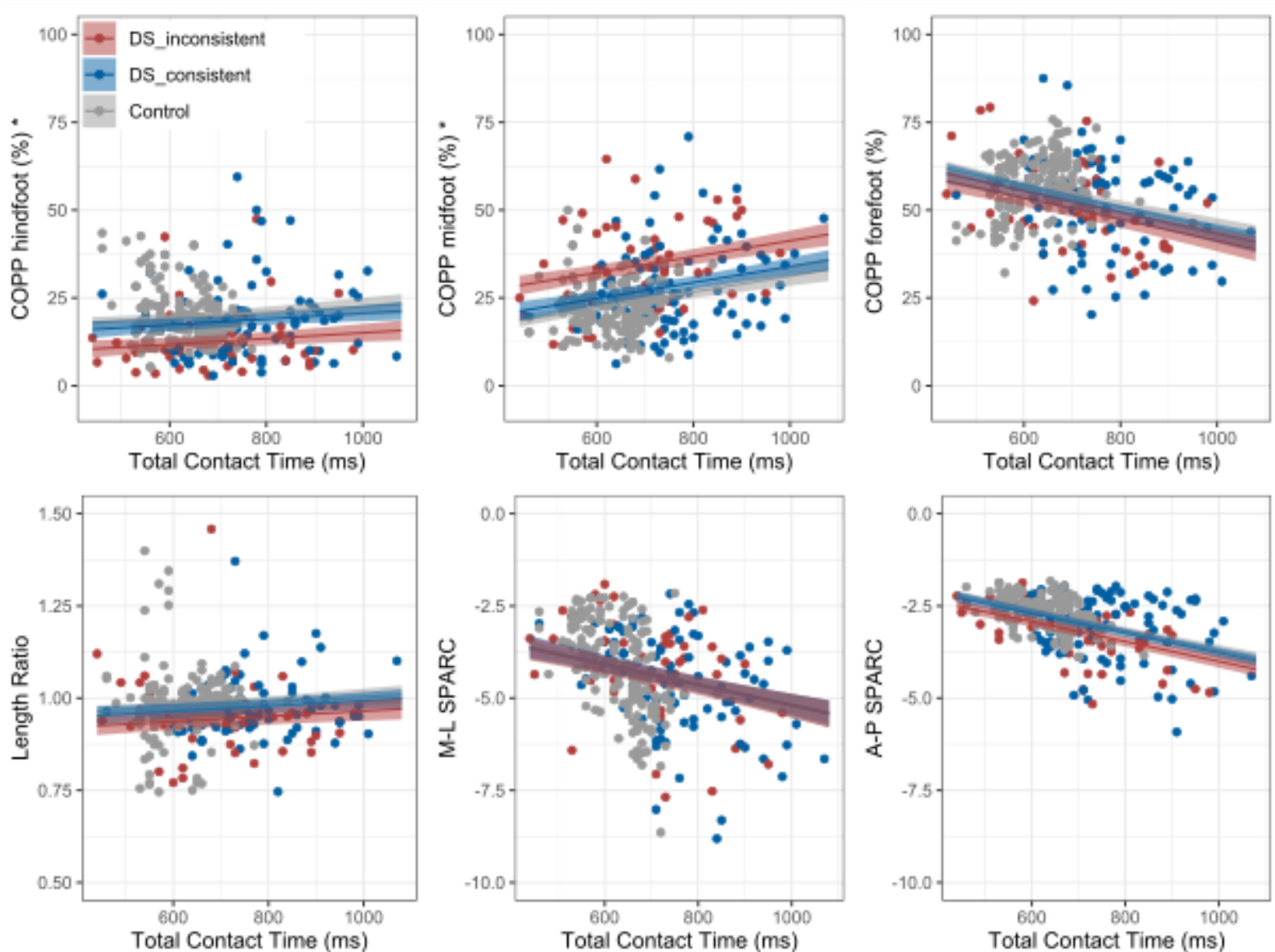

Figure B2: Linear mixed model predictions for total contact time within heel strikes. $*$ = Parameter with significant difference between heel strikes of the DS subgroups (dark red and blue) and control (grey) group. CoPP, center of pressure progression; SPARC, Spectral arc length; M-L, medio-lateral; A-P, antero-posterior. 